PROCEEDINGS OF THE

AMERICAN MATHEMATICAL SOCIETY

Volume 129, Number 11, Pages 3395-3399

S 0002-9939(01)06107-X

Article electronically published on April 25, 2001

\title{
A GENERALIZATION OF BENDIXSON'S CRITERION
}

\author{
MICHAL FEČKAN \\ (Communicated by Carmen C. Chicone)
}

\begin{abstract}
Bendixson's condition on the nonexistence of periodic solutions for planar ordinary differential equations is extended to higher dimensional ordinary differential equations with first integrals to preclude the existence of certain invariant Lipschitz compact submanifolds for those equations.
\end{abstract}

\section{INTRODUCTION}

Criteria of Bendixson and Dulac are well-known on the nonexistence of periodic solutions for planar ordinary differential equations [1, [6]. Extensions to higher dimensional cases are given by W.B. Demidowitsch [5], R.A. Smith [15], 16], S.N. Busenberg and P. van den Driessche [2, G. Butler, R. Schmid and P. Waltman [3], J.S. Muldowney [14, Y. Li and J.S. Muldowney [9, 10], and M.Y. Li and J.S. Muldowney [11, [12]. Conditions precluding the existence of nontrivial periodic orbits for mappings in $\mathbb{R}^{n}$ are given by C.C. McCluskey and J.S. Muldowney 13.

Stimulated by the papers [8]-14], the author of this note derived in 7] criteria on the nonexistence of certain invariant objects for finite dimensional dynamical systems. There are also studied dynamical systems on invariant submanifolds which naturally arise in differential equations possessing first integrals. It was already shown by W.B. Demidowitsch [5] that for a differential equation given by

$$
\dot{x}=f(x), \quad x \in \mathbb{R}^{n},
$$

and possessing a first integral with $n=3$, the Bendixson condition $\operatorname{div} f \neq 0$ in a simply connected region of a nondegenerate level set of the first integral precludes periodic solutions of (1.1) in the region. Unfortunately the Demidowitsch proof is incorrect and we present a correction here. This result was extended by M.Y. Li in his Ph.D. dissertation [8] in the context of former generalizations of Bendixson's condition proved by J.S. Muldowney [14].

We show in this note that if (1.1) has $p$ independent first integrals, the Bendixson criterion $\operatorname{div} f \neq 0$ implies the nonexistence of certain invariant $n-p$-1-dimensional objects of (1.1) on each nondegenerate level set of the first integrals. Similar results are proved in [12].

Received by the editors April 10, 2000.

2000 Mathematics Subject Classification. Primary 34A34, 34C40, 37C10.

Key words and phrases. Invariant submanifolds, first integrals, flows.

This work was supported by Grant GA-MS 1/6179/00. 


\section{Preliminaries}

We suppose that $f \in C^{1}\left(\mathbb{R}^{n}, \mathbb{R}^{n}\right)$. We need the following definition and result of 7].

Let $M \subset \mathbb{R}^{l}$ be an $m$-dimensional compact smooth orientable submanifold with a nonempty border $\partial M$ 17. Hence $\partial M$ is an $m-1$-dimensional compact smooth orientable submanifold. Of course, we always assume that $m \geq 2$.

Let $V \subset \mathbb{R}^{n}$ be a $k$-dimensional smooth submanifold of $\mathbb{R}^{n}$ with empty border $\partial V=\emptyset$.

Definition 2.1 ([7]). Let $\beta \in \operatorname{Lip}\left(M, \mathbb{R}^{n}\right)$ be such that $\beta(M) \subset V$ and $\tau=\beta / \partial M$ satisfy:

(I) $\tau$ is injective on $\partial M$.

(II) The inverse $\tau^{-1}: \tau(\partial M) \rightarrow \mathbb{R}^{l}$ is Lipschitz on the set $\tau(\partial M) \subset \mathbb{R}^{n}$.

We shall call the set $\mathcal{S}=\tau(\partial M)$ an $m-1-\mathbf{V}-\mathbf{L}-$ boundary of $V$. It is a generalization of smooth submanifolds of $V$.

Let $T_{v} V^{\perp}, v \in V$, be the orthogonal vector bundle to the tangent vector bundle $T_{v} V$ and let $Q_{v}$ be the orthogonal projection onto $T_{v} V^{\perp}$ in $\mathbb{R}^{n}$ with respect to the usual scalar product on $\mathbb{R}^{n}$. Let $N(v)$ be given by

$$
N(v)=Q_{v} D f(v) / T_{v} V^{\perp}: T_{v} V^{\perp} \rightarrow T_{v} V^{\perp} .
$$

We note that $\operatorname{div} f(v)=\operatorname{tr} D f(v)=\lambda_{1}+\lambda_{2}+\cdots+\lambda_{n}$, where $\lambda_{1}, \lambda_{2}, \cdots, \lambda_{n}$ are the eigenvalues of $D f(v)$.

Theorem 2.2 ([7]). If manifold $V$ is invariant for (1.1) and the inequality

$$
\operatorname{div} f(v)-\operatorname{tr} N(v) \neq 0
$$

holds for any $v \in V$, then there is no $k-1-V$-L-boundary $\mathcal{S}$ of $V$ which is invariant for (1.1).

\section{Systems With First integrals}

We assume the existence of $p$ first integrals $g_{1}, g_{2}, \cdots, g_{p} \in C^{2}\left(\mathbb{R}^{n}, \mathbb{R}\right)$ of (1.1). Let $0 \in \mathbb{R}^{p}$ be a regular value of the mapping $G=\left(g_{1}, g_{2}, \cdots, g_{p}\right)$. Hence $V=$ $G^{-1}(0)$ is a smooth $n$-p-dimensional submanifold of $\mathbb{R}^{n}$ without border.

We now show that condition (2.1) for this case can be simplified. Of course we have $\operatorname{tr} N(v)=\operatorname{tr} Q_{v} S(v) / T_{v} V^{\perp}$, where $S(v)=\left(D f(v)+D f(v)^{*}\right) / 2$.

Lemma 3.1. If $\lambda \neq 0$ is an eigenvalue of $Q_{v} S(v)$, then it is also an eigenvalue of $Q_{v} S(v) / T_{v} V^{\perp}$.

Proof. Let $Q_{v} S(v) w=\lambda w$ with $\lambda=\lambda_{1}+\imath \lambda_{2}, w=w_{1}+\imath w_{2}$. Then

$$
Q_{v} S(v) w_{1}=\lambda_{1} w_{1}-\lambda_{2} w_{2}, \quad Q_{v} S(v) w_{2}=\lambda_{1} w_{2}+\lambda_{2} w_{1} .
$$

If $w_{3} \in T_{v} V$, then we get

$$
\begin{aligned}
& \lambda_{1}\left\langle w_{1}, w_{3}\right\rangle-\lambda_{2}\left\langle w_{2}, w_{3}\right\rangle=0 \\
& \lambda_{1}\left\langle w_{2}, w_{3}\right\rangle+\lambda_{2}\left\langle w_{1}, w_{3}\right\rangle=0 .
\end{aligned}
$$

Since $\lambda \neq 0$ we get $\left\langle w_{1}, w_{3}\right\rangle=\left\langle w_{2}, w_{3}\right\rangle=0$, i.e. $w_{1}, w_{2} \in T_{v} V^{\perp}$. 
Lemma 3.1 implies that $\operatorname{tr} N(v)=\operatorname{tr} Q_{v} S(v)$. Now we compute $\operatorname{tr} Q_{v} S(v)$. Since $0 \in \mathbb{R}^{p}$ is a regular value of $G$, the vectors $\operatorname{grad} g_{i}(v), i=1,2, \cdots, p$, form a basis of $T_{v} V^{\perp}$. The vector $Q_{v} x=\sum_{i=1}^{p} c_{i} \operatorname{grad} g_{i}$ for an $x \in \mathbb{R}^{n}$ is determined by the equations

$$
\left\langle x-\sum_{i=1}^{p} c_{i} \operatorname{grad} g_{i}, \operatorname{grad} g_{j}\right\rangle=0, \quad j=1,2, \cdots, p .
$$

Let us put $e_{k}=(0,0, \cdots, 0,1,0, \cdots, 0)$, where 1 is on the $k$-th position. Then

$$
S(v) e_{k}=\frac{1}{2}\left(\frac{\partial f_{1}}{\partial x_{k}}+\frac{\partial f_{k}}{\partial x_{1}}, \frac{\partial f_{2}}{\partial x_{k}}+\frac{\partial f_{k}}{\partial x_{2}}, \cdots, \frac{\partial f_{n}}{\partial x_{k}}+\frac{\partial f_{k}}{\partial x_{n}}\right)
$$

and $Q_{v} S(v) e_{k}=\sum_{i=1}^{p} c_{k i} \operatorname{grad} g_{i}$ is determined by the equations

$$
\frac{1}{2} \sum_{s=1}^{n}\left(\frac{\partial f_{s}}{\partial x_{k}}+\frac{\partial f_{k}}{\partial x_{s}}\right) \frac{\partial g_{j}}{\partial x_{s}}=\sum_{i=1}^{p} c_{k i}\left\langle\operatorname{grad} g_{i}, \operatorname{grad} g_{j}\right\rangle, \quad j=1,2, \cdots, p .
$$

Consequently we get

$$
\operatorname{tr} Q_{v} S(v)=\sum_{k=1}^{p} \sum_{i=1}^{p} c_{k i} \frac{\partial g_{i}}{\partial x_{k}}
$$

The right-hand side of (3.2) has, according to (3.1), the form

$$
\frac{1}{2} \sum_{k=1}^{p} \sum_{i=1}^{p} A_{k i} \frac{\partial g_{i}}{\partial x_{k}} / B
$$

where

$$
\begin{aligned}
& A_{k i}= \\
& \operatorname{det}\left(\begin{array}{ccccc}
\left\langle\operatorname{grad} g_{1}, \operatorname{grad} g_{1}\right\rangle & \cdots & \sum_{s=1}^{n}\left(\frac{\partial f_{s}}{\partial x_{k}}+\frac{\partial f_{k}}{\partial x_{s}}\right) \frac{\partial g_{1}}{\partial x_{s}} & \cdots & \left\langle\operatorname{grad} g_{p}, \operatorname{grad} g_{1}\right\rangle \\
\vdots & \vdots & \vdots & \vdots & \vdots \\
\left\langle\operatorname{grad} g_{1}, \operatorname{grad} g_{p}\right\rangle & \cdots & \sum_{s=1}^{n}\left(\frac{\partial f_{s}}{\partial x_{k}}+\frac{\partial f_{k}}{\partial x_{s}}\right) \frac{\partial g_{p}}{\partial x_{s}} & \cdots & \left\langle\operatorname{grad} g_{p}, \operatorname{grad} g_{p}\right\rangle
\end{array}\right)
\end{aligned}
$$

and

$$
B=\operatorname{det}\left(\begin{array}{ccc}
\left\langle\operatorname{grad} g_{1}, \operatorname{grad} g_{1}\right\rangle & \cdots & \left\langle\operatorname{grad} g_{p}, \operatorname{grad} g_{1}\right\rangle \\
\vdots & \vdots & \vdots \\
\left\langle\operatorname{grad} g_{1}, \operatorname{grad} g_{p}\right\rangle & \cdots & \left\langle\operatorname{grad} g_{p}, \operatorname{grad} g_{p}\right\rangle
\end{array}\right) .
$$

By using the identities

$$
\sum_{s=1}^{n} \frac{\partial g_{j}}{\partial x_{s}} f_{s}=0, \quad j=1,2, \cdots, p,
$$

we get the new ones

$$
\sum_{k=1}^{n} \sum_{s=1}^{n} \frac{\partial g_{i}}{\partial x_{k}} \frac{\partial^{2} g_{j}}{\partial x_{k} \partial x_{s}} f_{s}+\frac{\partial g_{i}}{\partial x_{k}} \frac{\partial g_{j}}{\partial x_{s}} \frac{\partial f_{s}}{\partial x_{k}}=0, \quad i, j=1,2, \cdots, p .
$$


These identities imply

$$
\begin{gathered}
-\sum_{s=1}^{n} f_{s} \frac{\partial}{\partial x_{s}}\left\langle\operatorname{grad} g_{i}, \operatorname{grad} g_{j}\right\rangle=\sum_{s=1}^{n} \sum_{k=1}^{n}\left(\frac{\partial f_{s}}{\partial x_{k}}+\frac{\partial f_{k}}{\partial x_{s}}\right) \frac{\partial g_{i}}{\partial x_{k}} \frac{\partial g_{j}}{\partial x_{s}}, \\
i, j=1,2, \cdots, p .
\end{gathered}
$$

By using these equalities in the formulas for $A_{k i},(3.3)$ has the form

$$
\sum_{s=1}^{p} \sum_{i=1}^{p}-f_{s} B_{s i} / B
$$

where

$$
2 B_{s i}=\operatorname{det}\left(\begin{array}{ccccc}
\left\langle\operatorname{grad} g_{1}, \operatorname{grad} g_{1}\right\rangle & \cdots & \frac{\partial}{\partial x_{s}}\left\langle\operatorname{grad} g_{i}, \operatorname{grad} g_{1}\right\rangle & \cdots & \left\langle\operatorname{grad} g_{p}, \operatorname{grad} g_{1}\right\rangle \\
\vdots & \vdots & \vdots & \vdots & \vdots \\
\left\langle\operatorname{grad} g_{1}, \operatorname{grad} g_{p}\right\rangle & \cdots & \frac{\partial}{\partial x_{s}}\left\langle\operatorname{grad} g_{i}, \operatorname{grad} g_{p}\right\rangle & \cdots & \left\langle\operatorname{grad} g_{p}, \operatorname{grad} g_{p}\right\rangle
\end{array}\right) .
$$

Consequently (3.4) implies

$$
\operatorname{tr} N=-\langle f, \operatorname{grad} B\rangle /(2 B) .
$$

Furthermore, clearly the dynamics of (1.1) on $V$ are the same as for $\dot{x}=\alpha(x) f(x)$ for a positive $C^{1}$-smooth function $\alpha$ defined near $V$. Since

$$
\operatorname{div}(\alpha(x) f(x))=\alpha(x) \operatorname{div} f(x)+\langle\operatorname{grad} \alpha(x), f(x)\rangle,
$$

according to (3.5) condition (2.1) can be replaced by

$$
\alpha \operatorname{div} f+\langle\operatorname{grad} \alpha+\alpha W, f\rangle \neq 0
$$

on $V$, where $W=\operatorname{grad} B /(2 B)$.

Lemma 3.2. There is a $C^{1}$-smooth positive function $\alpha$ defined near $V$ such that $\langle\operatorname{grad} \alpha+\alpha W, f\rangle=0$ on $V$.

Proof. We have $\langle f, W\rangle=\frac{1}{2 B}\langle f, \operatorname{grad} B\rangle$ on $V$. Now by taking $\alpha=B^{-1 / 2}$ we get the result.

Summarizing, we have the following main result of this note.

Theorem 3.3. Let $g_{1}, g_{2}, \cdots, g_{p} \in C^{2}\left(\mathbb{R}^{n}, \mathbb{R}\right)$ be first integrals of (1.1). If $V=$ $G^{-1}(0)$ is a nondegenerate level set of the mapping $G=\left(g_{1}, g_{2}, \cdots, g_{p}\right)$ and in addition $\operatorname{div} f \neq 0$ on $V$, then there is no $n-p-1-V$-L-boundary $\mathcal{S}$ of $V$ which is invariant for (1.1).

Theorem 3.3 gives for $n=3$ the Demidowitsch result [5], but the proof of [5] is incorrect: formula (13) is not right. Furthermore, similar results are proved by M.Y. Li and J.S. Muldowney [8], 12], and the author was strongly motivated by [5], 8], [12].

Finally we note that clearly an open bounded subset of $V$, for $V$ from Theorem 3.3 , with a Lipschitz boundary is an $n-p-1-\mathrm{V}$-L-boundary of $V$. Furthermore, any minimally invariant closed set of (1.1) is lying on a level set of $G$. The minimality means that the invariant set does not contain a smaller nonempty invariant subset of (1.1). Consequently, Theorem 3.3 together with Sard's theorem [17] imply that (1.1) possessing $p$ first integrals of $C^{n-p+1}$-smoothness and satisfying $\operatorname{div} f \neq 0$ on $\mathbb{R}^{n}$ generically may have a minimally invariant object, which is an $r$-V-L-boundary of the nondegenerate level set $V$, with the dimension $r$ less than $n-p-1$. 


\section{REFERENCES}

1. I. BENDIXSON, Sur les curbes définiés par des équations différentielles, Acta Math. 24 (1901), 1-88.

2. S.N. BUSENBERG \& P. VAN DEN DRIESSCHE, A method for proving the nonexistence of limit cycles, J. Math. Anal. Appl. 172 (1993), 463-479. MR 94c:34039

3. G. BUTLER, R. SCHMID \& P. WALTMAN, Limiting the complexity of limit sets in selfregulating systems, J. Math. Anal. Appl. 147 (1990), 63-68. MR 91e:58152

4. W.A. COPPEL, "Stability and Asymptotic Behavior of Differential Equations", Health, Boston, 1965. MR 32:7875

5. W.B. DEMIDOWITSCH, Eine verallgemeinerung des kriteriums von Bendixson, Z. angew. Math. Mech. (ZAMM) 46 (1966), 145-146.

6. H. DULAC, Recherche des cycles limites, C. R. Acad. Sci. Paris 204 (1937), 1703-1706.

7. M. FEČKAN, Criteria on the nonexistence of invariant Lipschitz submanifolds for dynamical systems, J. Differential Equations (to appear).

8. M.Y. LI, Geometrical Studies on the Global Asymptotic Behaviour of Dissipative Dynamical Systems, Ph.D. Thesis, University of Alberta, 1993.

9. Y. LI \& J.S. MULDOWNEY, On Bendixson's criterion, J. Differential Equations 106 (1993), 27-39. MR 94j:34048

10. _ـ Evolution of surface functionals and differential equations, in "Ordinary and Delay Diff. Equations", J.K. Hale \& J. Wiener (Eds.), Pitman Res. Not. Math. Ser. Vol. 272, Longman, Harlow (1992), 144-148. CMP 97:08

11. M.Y. LI \& J.S. MULDOWNEY, Lower bounds for the Hausdorff dimension of attractors, J. Dyn. Diff. Equations 7 (1995), 457-469. MR 96f:58093

12. $\longrightarrow$ Dynamics of differential equations on invariant manifolds, J. Differential Equations (to appear).

13. C. C. McCLUSKEY \& J.S. MULDOWNEY, Bendixson-Dulac criteria for difference equations, J. Dyn. Diff. Equations 10 (1998), 567-575. MR 2000j:39008

14. J.S. MULDOWNEY, Compound matrices and ordinary differential equations, Rocky Mountain J. Math. 20 (1990), 857-872. MR 92h:15002

15. R.A. SMITH, An index theorem and Bendixson's negative criterion for certain differential equations of higher dimension, Proc. Royal Soc. Edinburgh Sect. A 91 (1981), 63-77. MR 83g:58059

16. R.A. SMITH, Some applications of Hausdorff dimension inequalities for ordinary differential equations, Proc. Royal Soc. Edinburgh Sect. A 104 (1986), 235-259. MR 88a:34056

17. M. SPIVAK, "Calculus on Manifolds", W.A. Benjamin, Inc., New York, 1965. MR 35:309

Department of Mathematical Analysis, Comenius University, Mlynská dolina, 84248 Bratislava, Slovakia

E-mail address: Michal.Feckan@fmph.uniba.sk 